\title{
Stressing the mind: The effect of cognitive load and articulatory suppression on attentional guidance from working memory
}

\author{
David Soto \\ Imperial College London, London, England \\ and University of Birmingham, Birmingham, England \\ AND \\ GLYN W. HuMPHREYS \\ University of Birmingham, Birmingham, England
}

\begin{abstract}
Four experiments explored the effect of cognitive load on the time course of top-down guidance of attention from working memory (WM). Observers had to search for a target presented among several distractors, with the target and distractor stimuli embedded inside different objects. On half of the trials, one of the distractor objects was cued by a matching item held in WM. When a single item was maintained in memory, search performance was impaired relative to a neutral baseline, where the memory and search displays did not match. These effects of WM on subsequent search were reduced by including a verbal suppression task during the WM and search displays, and by varying the WM load. The degree of competition for resources in WM is a key factor in determining the time course and magnitude of the interaction between WM and visual selection.
\end{abstract}

There is growing evidence that working memory (WM) and visual attention work in an interactive fashion. According to the biased competition model of selection (Desimone, 1998; Desimone \& Duncan, 1995), WM plays a key role in biasing attention to objects that are relevant for our goals and actions. Consistent with this, neurophysiological studies have shown that feedback from objects in WM can modulate early neuronal responses in visual cortex (i.e., V4) when a target that matches the memory representation has to be selected for a response (Chelazzi, Duncan, Miller, \& Desimone, 1998; Chelazzi, Miller, Duncan, \& Desimone, 1993). Recent behavioral studies have further shown that the deployment of attention in visual space can be automatically biased to stimuli that match the contents of WM. For example, Soto, Heinke, Humphreys, and Blanco (2005) asked observers to hold in WM an object presented at the beginning of each trial. After a short delay, a search display was presented with four different colored shapes, each containing a line. Participants had to identify the orientation of a tilted target line presented among vertical distractor lines, with each line appearing within a different colored shape. On some trials, one of these colored shapes could match the properties of the memory item (i.e., color, shape, or both). The matching object could contain either the target line (on valid trials) or a distractor (on invalid trials), and performance was assessed relative to a neutral baseline where the memory objects never re- appeared in the search display. Soto et al. (2005) found that when the WM item did reappear in the search display, there were effects on the time taken to detect the target and on the direction of the first saccade made in search. Relative to when the prime was neutral, reaction times (RTs) were slower, and fewer first saccades were made to the target when the memory item was invalid, whereas RTs were faster, and a higher proportion of first saccades went to the search target, when the WM stimulus was valid. Figure 1A illustrates the pattern of search latencies across the different conditions. In Soto et al.'s (2005) Experiment 4, there was never a valid match between the memory stimulus and the target line (i.e., the memory stimulus, when it appeared in the search display, was always invalid and surrounded a distractor line). Despite this, the appearance of the WM stimulus in the array still influenced the first saccade that took place and slowed search times relative to the neutral baseline (see also Soto, Humphreys, \& Heinke, 2006a, 2006b). Further, Soto and Humphreys (2006) have shown that matches between a stimulus in WM and a target can enhance visual awareness in parietal patients with visual extinction. The neuropsychological symptom of extinction arises when a patient can detect a single stimulus presented on the side of space contralateral to his or her lesion, whereas the patient fails to detect the same stimulus when it occurs simultaneously with an item on the unimpaired (ipsilesional) side (Karnath, 1988; Ward, Goodrich,

D.Soto, d.soto@imperial.ac.uk 
A

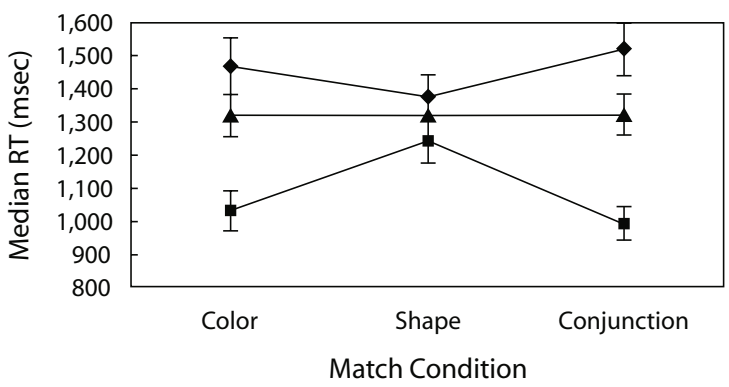

B

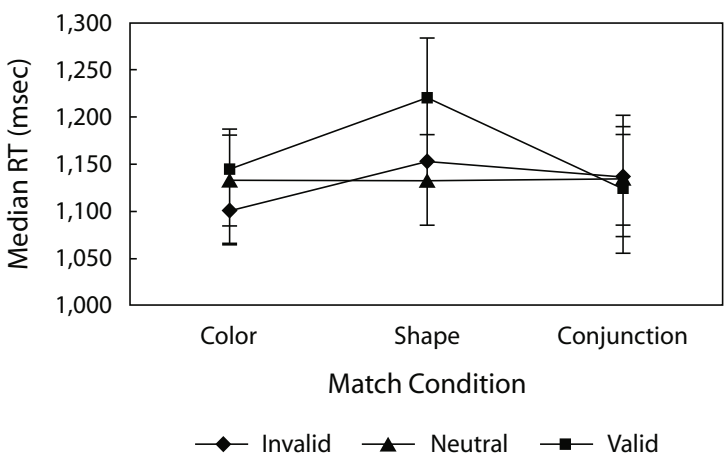

Figure 1. Search latencies as a function of the validity and match conditions: (A) prime held in WM; (B) prime passively registered. From "Early, Involuntary Top-Down Guidance of Attention From Working Memory," by D. Soto, D. Heinke, G. W. Humphreys, \& M. J. Blanco, Journal of Experimental Psychology: Human Perception \& Performance, 31, pp. 254-257. Copyright 2005 by the American Psychological Association. Reproduced with permission.

\& Driver, 1994). In such patients, extinction is reduced when the stimulus on the contralesional side matches the contents of WM. Importantly, neither the effects with normal participants nor those with the parietal patients were apparent when the same presentation conditions were used but participants were told not to commit the first items to memory. These data suggest that the effects are not due just to visual priming from the initial events and that, specifically, items committed to WM can influence early stages of visual selection. This is illustrated in Figure 1, which shows the pattern of search performance in Soto et al. (2005) when the prime did (A) and did not (B) have to be held in WM. Downing (2000) has shown similar effects of guidance of attention in search to an item matching the contents of WM.

In other studies, though, effects of WM on visual selection have not been observed. For example, Downing and Dodds (2004) showed participants two shapes, one of which was the target in a subsequent search display, and the other a memory stimulus that had to be remembered for a later report. They showed that the memory stimulus did not affect selection when it reappeared as a distractor in the search task (indeed, in their Experiment 2, search performance was even faster when the irrelevant memory prime was re-presented as a distractor in the search array, relative to when it was not re-presented). Woodman and Luck (2007) also reported similar findings. In their experiments, a to-be-remembered colored square was followed by a search display composed of several Landolt squares. Half the trials were invalid and the other half neutral. On invalid trials, a search distractor could match the color of the memory item, and on neutral trials, there was no match between the memory and search displays. Woodman and Luck did not find any evidence of attentional capture by the contents of WM (Experiments 1 and 2). Search latencies on invalid trials were similar to those on neutral trials. Interestingly, in line with Downing and Dodds, Woodman and Luck (Experiment 3) also found that when the WM load increased, attention could be directed away from distractors matching a memory item, so that reappearance of the WM stimulus in the search display improved search relative to the neutral baseline. This evidence suggests that, at least under certain conditions, information in WM that is irrelevant for current behavior can be shielded from the relevant task at hand, or it might even be used to guide search away from irrelevant distractors. Note that in this last case, participants must again keep any guidance from the WM stimulus separate (in this case, away from a distractor) from guidance from the task-relevant memory of the target (guiding search to the target). Soto et al. (2006a) presented some information that the frontal lobes were important for this shielding process. They examined conditions in which healthy participants showed biases in selection on the basis of the contents of WM. They found that frontal patients showed biases similar to controls' on the initial guidance of attention, but were then impaired at rejecting distractors that matched items held in WM. Soto et al. (2006a) suggested that frontal lobe regions are responsible for "compartmentalizing" information in WM (to guide search, on the one hand, and for later recognition, on the other). Because the memory representations for the target and the WM item were not kept separate, the patients had difficulties in rejecting WM items that were selected.

The apparent discrepancy between the above studies may be due to several factors. First, the cognitive load required by the memory task differed across the studies. In Downing and Dodds (2004) and Woodman and Luck (2007), WM loads of two or three items were used, whereas the studies showing positive effects of guidance from WM have used a single WM stimulus (Downing, 2000; Soto et al., 2005; Soto et al., 2006a, 2006b). It may be more critical to keep representations for the target and the WM stimuli separate when the WM load increases. Second, both Downing and Dodds and Woodman and Luck had observers perform an articulatory suppression task through the trial in order to prevent verbal recoding of the shapes in WM. This was not the case in studies showing positive guidance from WM (e.g., Soto et al., 2005; Soto et al., 2006b). Articulatory suppression may disrupt the maintenance of any item in WM (cf. Allen, Baddeley, \& Hitch, 2006), reducing the impact on search of stimuli held in WM (particularly with higher WM loads). Third, the interval between the memory item and the search display varies across experiments. Soto et al. (2005; Soto et al., 2006b) used a relatively short interval 
$(188 \mathrm{msec})$ between the offset of the memory prime and the presentation of the search array, whereas Woodman and Luck and Downing and Dodds used longer intervals ( 1 and $2 \mathrm{sec})$. It might be that memory effects on selection reported by Soto et al. (2005; Soto et al., 2006b) are determined by an initial and short-lasting strong activation of items as they are initially committed to memory. This effect may not be detected once items have been consolidated in WM.

Here, we present four experiments that assess factors that may be critical to the effects of WM on visual selection. In Experiment 1 we used the procedure of Soto et al. (2005) (see Figure 2), but varied the time between the offset of the previous WM item and the onset of the search display, to test whether the WM effect was confined to when there was a short interval between the WM item and the search task. In Experiment 2 we had participants carry out a verbal suppression task while the WM was presented. Does preventing the WM item from being translated into a phonological/articulatory form weaken its impact on subsequent visual selection? Experiment 3 evaluated the effect of WM load on search by requiring participants to hold two items rather than one item in WM. Experiment 4 combined the effects of WM load and articulatory suppression. The data provide important baseline parameters for the effects of WM on search.

\section{EXPERIMENT 1 \\ Temporal Course of the WM Guidance and Target Saliency}

The aim of this experiment was to examine the temporal course of the top-down guidance of attention through WM. This exploration of the time course of WM effects was carried out in combination with an examination of the effects of target saliency on performance. Previously, we have shown that an item held in WM can guide visual selection for highly salient (Soto et al., 2006b) as well as for less salient targets (Soto et al., 2005). In these prior studies, the search stimuli were oriented lines presented within colored shapes that could correspond to an item held in WM, and target saliency was varied by manipulating the orientation of the target relative to the distractors. The WM stimulus guided search even when the target was salient and "popped" out in a baseline search task (when there was no WM item and the number of distractors was varied; Soto et al., 2006b). Here, target saliency was varied in a similar way. If WM effects on search are strongest while the WM item is being consolidated, then top-down effects on search should be strongest when the interval between the WM item and the search display was short, and effects should weaken as the WM item is presented for longer. Any weakening of the effects of WM on search should be most pronounced when the search target is salient, when strong bottom-up cues compete for selection.

\section{Method}

Participants. The participants were 10 students from the School of Psychology at the University of Birmingham who were unaware of the purpose of the experiment. They were between 18 and 26 years of age, and their vision was normal or corrected to normal. They were given course credits or cash for their participation.

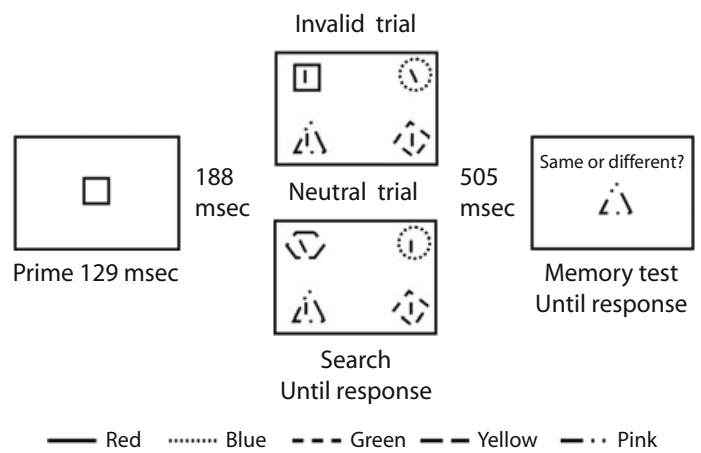

Figure 2. Examples of the display sequences in both conditions of prime validity.

Apparatus. The experiment was run on a Pentium IV computer with an ATI RAGE PRO 128-MB graphics card. The task was programmed with E-Prime (Version 1.0; Psychology Software Tools, 2002). The stimuli were displayed on a Samsung SyncMaster 753s color monitor. Monitor resolution was $1,024 \times 768$ pixels. Frame rate was fixed at $85 \mathrm{~Hz}$.

Task. A fixation display was presented at the beginning of each trial for $505 \mathrm{msec}$, which was followed by the memory item for $129 \mathrm{msec}$. The search display followed 188, 504, or 1,008 msec after the offset of the memory item. The shapes used were a circle $\left(1.8^{\circ} \times\right.$ $1.8^{\circ}$ of visual angle), a diamond $\left(1.91^{\circ} \times 1.91^{\circ}\right)$, a square $\left(1.5^{\circ} \times\right.$ $\left.1.5^{\circ}\right)$, a triangle $\left(2^{\circ} \times 1.5^{\circ}\right)$, and a hexagon $\left(2.38^{\circ} \times 0.95^{\circ}\right)$. The color of the objects could be red, green, blue, yellow, or pink, and the background was gray. Each object in the array was unique in color and shape. Four white lines were displayed inside a different object. Three of them were vertical, whereas the remaining one (the search target) was tilted $4.8^{\circ}$ or $38^{\circ}$ either to the left or the right. The length of the lines was $0.57^{\circ}$ and their width was $0.12^{\circ}$. The objects were arranged around an imaginary clock face of radius $6^{\circ}$. Each object could be at one of eight possible locations within the clock face: at 1, 2, 4, 5, 7, 8,10 , or 11 o'clock, each being positioned on any of the four possible $90^{\circ}$ quadrants (from 12 to 3 , from 3 to 6 , from 6 to 9 , and from 9 to 12 o'clock). Participants were instructed to memorize both the color and the shape of the memory item presented at the beginning of each trial and to discriminate the orientation of the target line. After the response for the search task, there was a memory test. A colored shape was displayed and the observers had to indicate whether it was identical or not to the memory item. Participants responded "same" in the memory test if both dimensions of the memory and probe object matched; otherwise they responded "different." On different trials, the memory and probe items could have just their color in common, just their shape in common, or neither attribute in common. Responses in the memory task were made by pressing one key for same and one for different stimuli. Participants were encouraged to perform well in both tasks. They were informed that only accuracy would be examined in the memory task and were asked to respond as accurately and as fast as possible in the search task.

Procedure. First, the observers were familiarized with the task and performed some practice trials; subsequently, they performed five blocks of 72 trials each. There were two different types of trials: In some, the memory stimulus was re-presented in the search array and in others it was not. On invalid trials, the memory stimulus was re-presented in the search display but always contained a distractor line rather than the target. On neutral trials, the memory stimulus was absent in the search array and none of the features of the memory stimulus were shared by any of the stimuli in the search array. Figure 2 illustrates examples of the different types of trials. Trial type was determined randomly, and each trial type had the same probability of occurrence. Participants were informed about these contingencies. 


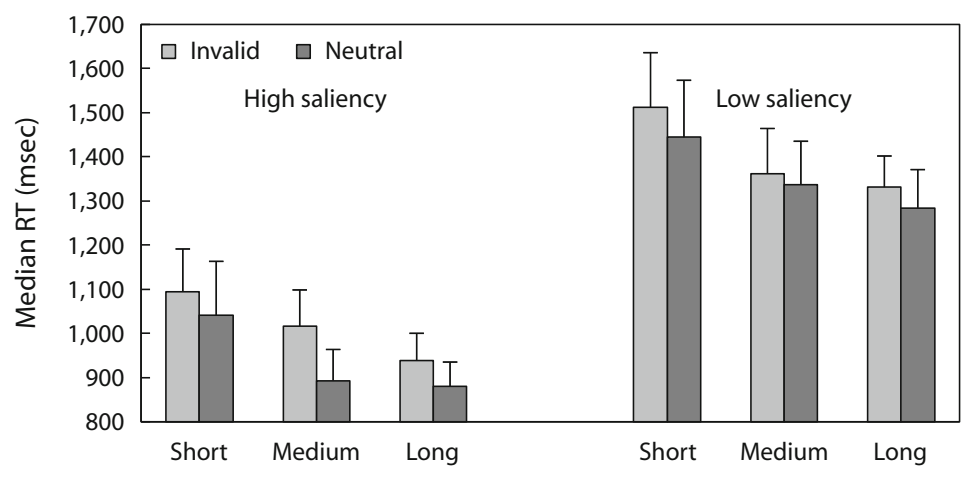

ISI

Figure 3. Median RTs as a function of the saliency of the target, the validity of the memory item, and the time interval between the memory and search displays.

\section{Results}

Errors in the search task were minimal (1\%) and were not analyzed further. Performance in the memory test was good (mean 4\% error). In all of the experiments reported in this article, search RTs were analyzed only for trials on which both responses were correct, using the median for each participant in each condition. In the following ANOVAs, all $p$ values for main effects or interactions were computed using the conservative GreenhouseGeisser method with corrected degrees of freedom (though, for the sake of clarity, the original degrees of freedom are reported).

First, we conducted a 2 (validity) $\times 2$ (target saliency) $\times$ 3 (ISI) ANOVA. This showed slower performance on invalid trials $(1,209 \mathrm{msec})$ than on neutral trials $(1,146 \mathrm{msec})$ $[F(1,9)=11.23, p<.008]$ and slower performance with low- than with high-saliency targets $(1,378 \mathrm{vs} .977 \mathrm{msec})$ $[F(1,9)=45.78, p<.0001]$. There was also an effect of ISI $[F(2,18)=6.69, p<.025]$, with faster performance as the ISI increased $(1,272,1,152$, and $1,108 \mathrm{msec}$ for the short, medium, and long ISIs, respectively). These effects were independent of one another. The effect of validity did not rely on the ISI $[F(2,18)=0.14, p<.86]$ or on the saliency of the target $[F(1,9)=3.29, p<.1]$. The threeway interaction was also unreliable $[F(2,18)=1.72, p<$ .21; see Figure 3].

We also assessed whether WM content affected the fastest search performance, as an index of early modulation of search. According to some views, any top-down effects on visual selection take place rather late in any stream of processing (e.g., Theeuwes, Reimann, \& Mortier, 2006), and hence may not be expected to occur on the fastest RTs in a study. Contrary to this, we have previously found effects of the WM stimulus even on the fastest trials in search tasks (Soto et al., 2005; Soto et al., 2006b). To examine this here, we divided the distribution of RTs for the different conditions into two bins containing just the fastest RTs up to, respectively, the 5 th and the 10 th percentiles, in order to test whether effects emerged on the fastest responses (see Soto et al., 2005; Soto et al., 2006b). The results were similar to those noted above (see the Appendix).

\section{Discussion}

We found that there was an effect of the item held in WM on search. RTs were slowed when the WM reoccurred and contained a distractor line (on invalid trials) in comparison with when the WM stimulus did not reoccur in the search display. This held even when the fastest RTs in each condition were examined (see the Appendix). Using identical stimuli (i.e., same size, color, luminance, display durations, etc.), Soto et al. (2005) found that there was no effect of the first stimulus when participants did not have to maintain it in memory (see Figure 1), indicating that the effects were not simply due to bottom-up priming from the initial stimulus ${ }^{1}$ (see also Downing, 2000; Soto \& Humphreys, 2006; Soto et al., 2006a, all of whom have failed to find bottom-up priming on subsequent search when the prime did not have to be committed to memory). Instead, the findings indicate that an item in WM can influence search even for a salient target and even when the contents of WM never cue the search target (given that we only used invalid and neutral conditions).

The effects of memory guidance were not modulated by either the saliency of the target or the time interval between the memory item and search, and there was no evidence for WM effects to decrease as the interval increased even when target lines were salient. This is in agreement with our previous observation that WM contents can guide visual selection even when highly salient bottom-up cues are present (Soto et al., 2006b). The present results extend this finding to show that memory effects with salient targets are not confined to a period when the initial item is being consolidated in WM, but they arise even when the interval is long enough to make it highly likely that memory consolidation has occurred. There was nevertheless an effect of the ISI on overall speed of performance, with response latencies being faster as the ISI between memory and search displays increased. This may reflect faster disengagement of attention from the memory item to the search task, once WM was consolidated; crucially, this did not affect the subsequent guidance of selection.

The additivity of the cuing effects with the ISI here are also of interest because they suggest that the results are 
also not due to the persistence of some form of perceptual representation of the prime (cf. Coltheart, 1980, 1983), which then guides search to a matching item in the search display. Any perceptual memory is likely to have decayed at the longer intervals examined here (504 and 1,008 $\mathrm{msec}$ ). Note also that transient perceptual memories are conceived of as being formed in a relatively passive fashion, and so should affect performance even when participants are not required to hold the stimulus in WM. However, as we have noted, there is considerable evidence that mere presentation of the prime is not sufficient to generate the present effects (Downing, 2000; Soto et al., 2005; Soto \& Humphreys, 2007; Soto et al., 2006a, 2006b).

\section{EXPERIMENT 2 Effects of Articulatory Suppression}

In prior studies of the effects of WM on visual selection, participants have been asked to carry out verbal suppression of the WM item prior to the presentation of a subsequent search display (Downing, 2000; Downing \& Dodds, 2004). This has been done in order to prevent participants from verbally recoding the visual information (Baddeley, 1986), to ensure that any effects stemmed from a visual memory representation. According to the influential model of WM suggested by Baddeley (1992, 2003), WM comprises a control system with a number of separate components, including a central executive, a phonological loop, and a visuospatial sketchpad. The function of the phonological loop is to hold phonological memory traces briefly before they fade, with these representations being refreshed by rearticulation. When articulatory suppression is carried out, visual representations of stimuli are less likely to be recoded into a phonological form (Baddeley, 2003). However, it may be that at least part of the effect of WM on selection comes from a match between a search display and a verbal WM representation (Soto \& Humphreys, 2007), so that the effect of WM on selection may be reduced when articulatory suppression is conducted. Articulatory suppression may also reduce WM effects on selection if suppression also (at least partially) reduces the capacity available for WM. In our prior studies, articulatory suppression was not required. Here, we examined the consequences of introducing articulatory suppression on the guidance of visual selection from the WM item.

\section{Method}

Participants. Fifteen new students took part, from the same pool as was used for Experiment 1.

Apparatus. The apparatus was the same as was used in Experiment 1.

Task and Procedure. There were two differences between this and the previous experiment. A first difference is that here we only used a low-saliency target. A second difference is that we included an articulatory suppression task prior to the presentation of the memory item. In the present study, two different numbers, selected randomly from 1 to 9 , were displayed at fixation for $505 \mathrm{msec}$ and followed by a blank interval for $952 \mathrm{msec}$ before the memory item appeared (using the same timing conditions as in Experiment 1). Participants were instructed to repeat both numbers aloud at a rate of 2 repetitions/sec, approximately, and at least until the search task was performed. The experimenter was present through the session in order to ensure that participants performed as instructed. In this experiment, we only used a low-saliency target.

\section{Results}

Errors in the search task were minimal (1\%) and were not analyzed further. Performance in the memory task was good $(92 \%$ correct).

A $2 \times 3$ repeated measures ANOVA with validity and ISI as factors was carried out on median RTs. The effect of validity was significant $[F(1,14)=16.24, p<.001]$ : Performance was slower on invalid trials $(1,049 \mathrm{msec})$ than on neutral trials $(991 \mathrm{msec})$. The effect of validity interacted with ISI $[F(2,28)=4.92, p<.025]$. Additional ANOVAs with validity as a factor were carried out at each ISI. The effect of the memory distractor on search was not reliable at the shortest and medium ISIs $(p<.18$ and $p<$ .16 , respectively), but the effect was much stronger and highly reliable at the longest ISI $(p<.0001)$. This pattern is depicted in Figure 4. Analyses of the fastest RTs (up to the 5 th and 10th percentiles) failed to show any effects of prime validity on performance (see the Appendix).

\section{Discussion}

The presence of a secondary articulation task had an impact on the time course over which WM influenced visual selection. Under conditions of articulatory suppression, there was relatively little effect of memory guidance at the shortest and medium intervals between the memory item and the search task, but an effect did emerge at the longest interval. The effects were also not present at the fastest RTs (see the Appendix). The present data contrast with those reported in Experiment 1 and with data from our previous studies, in which strong effects of memory guidance have been consistently observed at the shortest ISI used here (Soto et al., 2005; Soto et al., 2006b). Given that the only difference between the studies was the inclusion of a verbal suppression task, it appears that the execution of the verbal suppression task influences the effectiveness with which WM interacts with visual

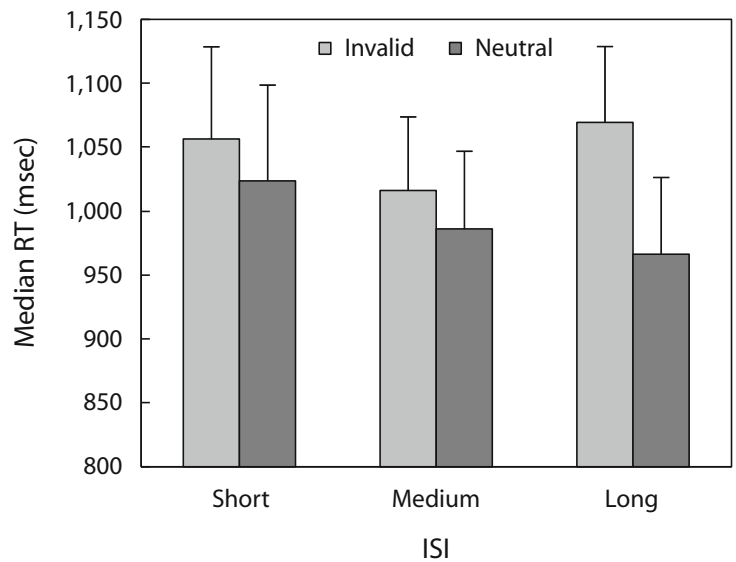

Figure 4. Median RTs as a function of the validity of the memory item and the time interval between the memory and search displays. 
attention; in particular, articulatory suppression appears to slow down the time course of the WM effect on visual selection. This effect could come about in either of two ways. One possibility is that the suppression task reduces the likelihood that the WM is encoded phonologically at the short and medium ISIs, and phonological recoding is important for at least supporting the effects of WM on selection. At the longer ISI, there is a greater likelihood of the WM being coded phonologically, despite the articulatory suppression task, and hence a top-down effect on selection then emerges. An alternative possibility is that the suppression task reduces the resources available to support WM, so that few resources are therefore available to direct visual attention to an item in the search display matching the WM stimulus. However, because the item in WM is consolidated over time, some resources may be released to support WM-based guidance of selection. Whichever is the case, we note that prior studies showing an effect of positive guidance from WM on selection, even when participants undergo articulatory suppression when the WM stimulus is exposed, have tended to use relatively long intervals (Downing, 2000), consistent with the present results. These data also go against accounts of the findings based on automatic visual priming or based on a persistent perceptual representation of the memory prime. If either automatic bottom-up priming or perceptual memories were crucial to generate positive attentional guidance, we would have expected effects on search regardless of the ISI and regardless of the presence of articulatory suppression. Clearly this was not the case. By elimination, these data add support to a WM-based account of performance. In line with this, if it is crucial that sufficient processing resources are available to support WM-based guidance of attention for top-down effects to emerge, then it is also possible that any WM effects will be modulated by introducing a greater WM load. We did this in Experiment 3.

\section{EXPERIMENT 3 Effects of Increased Memory Load}

\section{Method}

Participants. Fourteen new students took part, drawn from the same pool as in Experiments 1 and 2.

Apparatus. The apparatus was the same as in Experiment 1.

Procedure. The procedure was identical to that used in Experiment 1 except that here only low-saliency targets were used and observers were required to keep two objects in memory (instead of just one, as in Experiment 1). The two memory items differed in both color and shape. In order to keep the four objects in the search display also unique in color and shape, we added a new possible shape (a hexagon rotated $90^{\circ}$ ) and a new color (black) to the set of items that could appear. On invalid trials, either of the memory objects could reappear as a distractor in the search display. This occurred on half the trials; on the remaining (neutral) trials, the WM did not reappear in the search display. After the search task was completed, two stimuli were presented as memory probes. On same object trials, the two probes were identical to the original WM stimuli. On different object trials, one of the probes could differ either in color or shape from the original, whereas the other stimulus was the same. Participants had to press one of two keys, depending on whether the probes were the same as or different from the memory items.

\section{Results}

Errors in the search task were minimal (3\%). Performance in the memory task was good $(76 \%)$. We carried out a $2 \times 3$ ANOVA over the median RTs with validity and ISI as factors. Performance was slower on invalid trials $(1,101 \mathrm{msec})$ than on neutral trials $(1,015 \mathrm{msec})$ $[F(1,13)=11.5, p<.005]$. There was also a main effect of ISI $[F(2,26)=12.26, p<.001]$, with the slowest performance at the shortest ISI relative to the medium and longest ISIs ( $p<.004$ and $p<.001$, respectively). These two factors did not interact $(F<1)$. Figure 5 depicts this pattern of results. As in Experiment 1, there were effects of prime validity on the fastest RTs (see the Appendix).

In order to test whether the increased memory load reduced the effect of memory on selection, we compared performance across Experiments 1 (one memory item) and 3 (two memory items). The effect of validity did not interact with the experiment $[F(1,22)=1.04, p<.24]$. The three-way interaction between ISI, experiment, and validity was also not significant $[F(2,44)=1.03, p<$ .35]. This null result suggests that increasing the memory load from one to two items does not have a strong impact on the top-down bias of attention from WM.

\section{Discussion}

The data from Experiment 3 largely replicate those from Experiment 1. There were reliable effects of WM guidance on visual selection, and these effects occurred across the 3 ISIs used. There was no diminution of the effects across these two experiments, indicating that memory-based guidance was as strong from two WM stimuli (in Experiment 3) as it was from a single item (in Experiment 1). It is of course possible that WM effects would decrease if the WM load was increased further, but at least within these capacity limits, the guidance effects were robust. This does suggest, though, that verbal recoding of the stimuli is unlikely to be necessary to generate a top-down effect, given that it is very unlikely that partici-

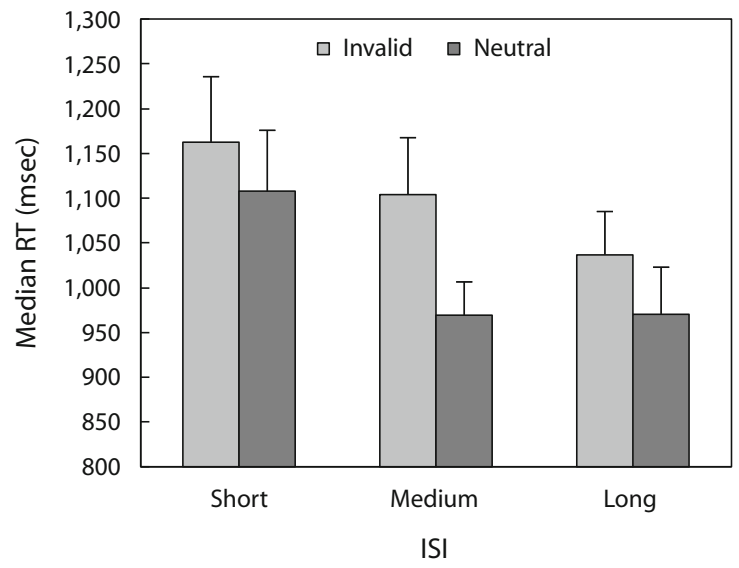

Figure 5. Median RTs as a function of the validity of the memory item and the time interval between the memory and search displays. 
pants would be able to name both the shapes and both the colors at the shortest ISI.

\section{EXPERIMENT 4 \\ Increased Cognitive Load Plus Verbal Suppression}

In the final study, we examined the combined effects of WM load and articulatory suppression on top-down guidance effects from WM. Does articulatory suppression produce an additional reduction in the effects of WM on subsequent search when WM capacity is subject to greater challenge (in Experiment 3 in comparison with Experiment 4)?

\section{Method}

Participants. Fourteen new students took part, from the same pool as for Experiments 1-3.

Apparatus. The apparatus matched that in Experiment 1.

Procedure. The procedure was an amalgamation of the procedures used in Experiments 2 and 3. As in Experiment 2, participants were cued to initiate articulatory suppression of two digits prior to the WM display, and they were asked to continue articulatory suppression up to when the search display appeared. As in Experiment 3, two WM items were presented, both initially and at test.

\section{Results and Discussion}

Errors in the search task were minimal $(2 \%)$ and were not analyzed further. Performance in the memory task was good $(80 \%)$. We carried out a $2 \times 3$ ANOVA over the median RTs with validity and ISI as factors. Performance did not differ between invalid trials $(980 \mathrm{msec})$ and neutral trials $(954 \mathrm{msec})[F(1,13)=3.68, p<.077]$. The main effect of ISI was significant $[F(2,26)=4.89, p<.031]$, with slowest performance in the shortest and medium ISIs (940 and $944 \mathrm{msec})$ in comparison with the longest one $(1,015 \mathrm{msec})(p<.02$ and $p<.045$, respectively). These two factors did not interact. This is illustrated in Figure 6. Given that the main effect of validity was not reliable overall, we did not analyze performance at the fastest end of the response distribution.

The data from Experiment 4 were compared with those from Experiment 2 in order to assess the effect of increasing the WM load on performance. A 2 (experiment) $\times 2$ (validity) $\times 3$ (ISI) ANOVA was carried out. There were significant effects of ISI and validity $[F(2,54)=6.31, p<$ .008 , and $F(1,27)=17.65, p<.0001$, respectively]. Interestingly, the effect of validity across the different ISIs varied as a function of the WM load, as indexed by a reliable three-way interaction between ISI, validity, and experiment $[F(2,54)=3.57, p<.038]$. In order to identify the source of this interaction, we computed the costs on performance (invalid RT - neutral RT) across each ISI in each experiment and then performed independent $t$ tests to assess where differences emerged across the experiments. There were no differences in the size of the costs across experiments when the shortest and the medium ISIs were considered $[t(27)=0.32, p<.75$, and $t(27)=0.55, p<$ .59 , respectively]. However, a difference emerged at the longest ISI $[t(27)=3.53, p<.001]$, with the size of the costs being bigger with low load (Experiment 2) than with high load (Experiment 4). A similar comparison between

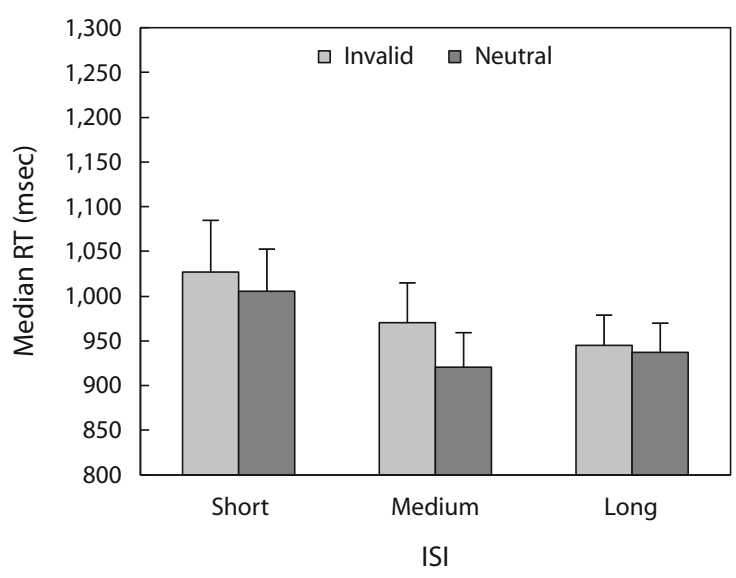

Figure 6. Median RTs as a function of the validity of the memory item and the time interval between the memory and search displays.

the data from Experiments 3 and 4 (same memory load, but with articulatory suppression introduced for Experiment 4) showed a significant interaction between experiment and validity $[F(1,26)=4.25, p<.049]$. The costs from attention being guided to the irrelevant memory distractor were reduced with verbal suppression (in Experiment 4) relative to when there was no verbal suppression (in Experiment 3).

\section{Memory Accuracy Data Across Experiments}

We also conducted an overall analysis of memory performance across the different experiments. A 4 (experiment $) \times 3$ (ISI) $\times 2$ (validity) ANOVA over the percentage of correct responses gave a reliable effect of experiment $[F(1,49)=25.99, p<.0001]$. The worst memory performance was found in Experiments 3 and 4, compared with Experiments 1 and $2(p<.0001)$. Memory performance did not differ between Experiments 1 and $2(p<.326)$, nor did it differ between Experiments 3 and $4(p<.16)$. These data clearly show that memory performance was affected by cognitive load. Memory accuracy was similar when the memory item reappeared in the search display as a distractor, compared with when it was absent $[F(1,49)<1]$. Thus, memory performance did not appear to be modulated by the validity of the memory item (though see Woodman \& Luck, 2007). This pattern held when only the data from Experiments 3 and 4 were considered, where performance was far from being at ceiling $[F(1,13)<1$ for both experiments]. Table 1 illustrates the pattern of memory performance across the different validity conditions of the different experiments.

\section{Discussion}

In the present experiment, the observers were asked to hold two objects in memory while performing an articulatory suppression task. Under these conditions, the effects of WM on selection decreased relative to when articulatory suppression was conducted with just a single WM item (Experiment 2) and relative to when there 
Table 1

Mean Proportion of Correct Memory Responses As a Function of the Validity and ISI Factors Across the Different Experiments

\begin{tabular}{|c|c|c|c|c|c|c|c|c|c|c|c|c|c|c|c|c|}
\hline & \multicolumn{4}{|c|}{ Experiment 1} & \multicolumn{4}{|c|}{ Experiment 2} & \multicolumn{4}{|c|}{ Experiment 3} & \multicolumn{4}{|c|}{ Experiment 4} \\
\hline & \multicolumn{2}{|c|}{ Invalid } & \multicolumn{2}{|c|}{ Neutral } & \multicolumn{2}{|c|}{ Invalid } & \multicolumn{2}{|c|}{ Neutral } & \multicolumn{2}{|c|}{ Invalid } & \multicolumn{2}{|c|}{ Neutral } & \multicolumn{2}{|c|}{ Invalid } & \multicolumn{2}{|c|}{ Neutral } \\
\hline & $M$ & $\overline{S E}$ & $M$ & $\overline{S E}$ & $M$ & $\overline{S E}$ & $M$ & $\overline{S E}$ & $M$ & $\overline{S E}$ & $M$ & $\overline{S E}$ & $M$ & $\overline{S E}$ & $M$ & $S E$ \\
\hline Short ISI & .96 & .03 & .95 & .03 & .93 & .02 & .92 & .02 & .78 & .02 & .78 & .02 & .72 & .02 & .72 & .02 \\
\hline Medium ISI & .95 & .03 & .94 & .02 & .94 & .02 & .92 & .02 & .80 & .02 & .82 & .02 & .77 & .02 & .81 & .02 \\
\hline Long ISI & .96 & .03 & .96 & .03 & .94 & .02 & .92 & .03 & .82 & .02 & .80 & .03 & .81 & .02 & .76 & .03 \\
\hline
\end{tabular}

was a memory load of two items but no articulatory suppression (Experiment 3). These data again go against accounts of the effects in terms of bottom-up priming and/ or persistent perceptual memories for stimuli, since both priming and perceptual memories should influence performance regardless of whether articulatory suppression is introduced. The present results, though, confirm that articulatory suppression can reduce WM-based guidance of visual attention, and this is particularly the case when the memory load increases. We discuss below how this effect can arise.

\section{GENERAL DISCUSSION}

The main findings can be summarized as follows:

1. Under conditions in which a single item was maintained in WM, there was reliable attentional guidance from WM across the entire range of ISIs and regardless of the saliency of the target (Experiment 1). This result matches previous evidence indicating an involuntary bias on subsequent visual selection from items held in WM (Soto et al., 2005; Soto et al., 2006a, 2006b). However, the effects of memory guidance were reduced when articulatory suppression was carried out (Experiments 2 and 4). Here, memory effects on search were only evident with a small load and at the longest ISI.

2. Effects of increasing the memory load itself were assessed in Experiment 3. Increasing the memory load from one to two items did not alter the effects of the WM representation on selection, and the pattern of the data matched that from Experiment 1 (WM load of one). However, the WM effect on selection was eliminated (even at the long ISI) when participants undertook articulatory suppression when two items had to be held in WM. Thus, articulatory suppression and WM load combined to modulate the interaction between WM and visual attention.

3. Our analysis of memory performance in the experiments provides confirmatory evidence that it became more difficult to consolidate and maintain the WM stimulus as the load increased (i.e., in Experiments 3 and 4 in comparison with Experiments 1 and 2; see Jolicœur \& Dell'Acqua, 1998; Vogel, Woodman, \& Luck, 2006). Memory performance was not influenced by the reappearance of the memory item, even when the capacity of WM was stressed (i.e., in Experiments 3 and 4).

Previous research has shown that there can be an early, involuntary, top-down directing of attention to stimuli matching the contents of WM (Olivers, Meijer, \& Theeuwes, 2006; Soto et al., 2005; Soto \& Humphreys, 2006;
Soto et al., 2006a, 2006b). In the studies of Soto et al., a match between an item held in WM and a subsequent search display was shown to affect the direction of the first saccade and to slow down even the fastest responses' latencies to the search target. Furthermore, these effects have been established even when the WM item is always invalid when it reappears in the search display (i.e., when it always surrounds a distractor rather than a target). In contrast, Downing and Dodds (2004) and Woodman and Luck (2007) have reported the reverse effect, with RTs being speeded when the WM item matches the target. This suggests that attention can be directed away from rather than being drawn toward stimuli matching the information held in WM. There are several differences between the studies, though, and in the present study we examined some of the potentially critical factors.

One difference between the studies relates to the time interval between the memory item and the subsequent search display. Soto et al. (2005; Soto et al., 2006b) used a relatively short interval between the stimuli, and any effects under these circumstances might reflect the ongoing consolidation of the WM stimulus coinciding with the presentation of the search display. In contrast, studies in which no or reversed WM effects have been observed have typically used longer intervals between the presentation of the WM stimulus and the search display (Downing \& Dodds, 2004; Woodman \& Luck, 2007). We examined the effects of the interval between the WM and search stimuli in Experiment 1 and established a reliable influence of WM on visual selection across a range of ISIs (see also Experiment 3). The effects of WM on selection do not seem to be confined to instances in which consolidation processes that afford consistent and durable representations in WM may still be operating.

Another important difference between the studies is that, in instances in which no or reversed WM effects have been observed on selection, participants have been required to carry out articulatory suppression while the WM stimulus is present. In our previous studies (Soto et al., 2005; Soto et al., 2006b), articulatory suppression has not been required. In Experiments 2 and 4, we introduced articulatory suppression across the exposure of the WM and search stimuli. Under this condition, there was a decreased effect of the WM item on selection, with a reliable effect emerging only at the longest ISI in Experiment 2 (see also Downing, 2000, for a similar finding). The memory effects on selection were indeed abolished in Experiment 4, likely reflecting the combined effects of articulatory suppression and increased WM load. This 
result may occur because the guidance of attention from WM may benefit when the memory representation is sustained through verbal coding, and the likelihood of verbal recoding taking place is reduced under articulatory suppression (Baddeley, 1986). The importance of verbal coding for WM-based guidance of visual selection is supported by a recent study of Soto and Humphreys (2007), in which strong effects of memory-based guidance were demonstrated even when the WM stimulus comprised a verbal description (i.e., "Red Square"). Other evidence points to search also being affected by semantic information associated with the expected target (Moores, Laiti, \& Chelazzi, 2003). The means by which relatively abstract verbal and semantic information has an impact on visual selection remains a question for future research, though given the ISIs used in Soto and Humphreys (2007), it seems unlikely that the effects were modulated by explicit visual imagery. Similarly, in Moores et al., effects were due to an item associated with the target, not the target itself, and it is improbable that participants imagined any associate when cued to search for a given target on a trial. Nevertheless, other research indicates that verbalization is not always sufficient for top-down effects to occur. For example, Soto and Humphreys (2006) showed that in neuropsychological patients, the phenomenon of extinction was reduced when the stimulus on the contralesional side matched with an item being held in WM. However, there was no effect on extinction when patients verbalized but did not hold the initial item in WM. In this case, verbalization alone was not sufficient for the initial item to guide search. We suggest, then, that verbal and visual (and perhaps even semantic) representations may each be used (and indeed may be mutually supportive) in directing visual attention. For example, holding a representation in verbal WM may automatically preactivate related visual attributes, which then direct visual attention to a matching item in the search display. This verbally mediated preactivation may be less effective than preactivation from a visual memory representation, though, and indeed was ineffective in our neuropsychological study (Soto \& Humphreys, 2006).

However, articulatory suppression may not just interfere with WM-based attentional guidance by limiting the amount of verbal coding taking place and how well items are coded in WM. In addition, articulatory suppression may consume part of the resources available to support top-down effects on selection. For example, according to one account of top-down effects on search, the biased competition model (Duncan, Humphreys, \& Ward, 1997), a WM representation in prefrontal cortex may feed back to provide temporary preactivation of corresponding representations in temporal cortex, and this activation modulates subsequent target selection. Now, if this feedback is weakened when resources are constrained, there may be a dissociation, with the continued maintenance of an item in prefrontal cortex and reduced activation in temporal cortex - the result being that an item can be remembered but no longer influences visual selection. In line with this argument, we found that WM effects on selection were reduced in Experiments 2 and 4 despite the WM item being consolidated (i.e., search was always examined on trials in which recognition memory was correct). This strongly argues against the proposal that continued consolidation of the WM item leads to effects of WM on selection. Rather, these findings suggest that, at least under conditions in which the availability of resources is stressed, WM can be "compartmentalized" or "divided" according to the most critical stimulus for the current task goal. Under load, it may be most critical to separate memory and search information, to maximize resources allocated to WM and search tasks specifically. If the information in WM can be isolated from the search stimuli, then topdown effects on selection would decrease (see Downing \& Dodds, 2004; Soto et al., 2006a, for neuropsychological evidence about the necessary role of the frontal cortex in compartmentalizing WM). If the irrelevant stimulus in WM is suppressed, to maximize the separation from the target's representation, then search might be guided away from any representation of the WM stimulus in the search display, as reported by Downing and Dodds and by Woodman and Luck (2007) under high-load conditions. On the other hand, under low cognitive demands, there might be enough spared resources to feed back information held in WM (i.e., in prefrontal areas) to early visual areas - the result being mandatory, automatic attentional capture to a memory-matching stimulus.

We conclude that articulatory suppression modulates the top-down guidance of attention (1) by reducing the likelihood of verbal recoding taking place and (2) by consuming resources needed to support top-down effects on selection. Unlike Downing and Dodds (2004) and Woodman and Luck (2007), though, we failed to find any evidence for a reversed effect of WM on selection (with attention being directed away from the WM stimulus in the search display). Indeed, our use of just invalid and neutral trials should have encouraged participants to not attend to the WM stimulus in the search display. Again, this did not hold. The contrasting results also cannot be attributed to differences in the type of match taking place between WM and the search display. Woodman and Luck had matches based on the color of the items, whereas Downing and Dodds had items match on shape. We had items match on both counts, and whereas color was more generally effective (see also Soto et al., 2005), effects of both attributes in attracting attention were observed. Hence the precise reasons for the contrasting results remain unclear. Nevertheless, the present studies show that WM effects do tend to be reduced when articulatory suppression is added and the memory load increased, providing important boundary constraints on top-down guidance of visual selection. The presence of articulatory suppression in these other studies would at least have weakened the impact of WM on search.

A final point is that the present data also shed light on the automaticity of top-down guidance of attention from WM. Although participants found it difficult to avoid attending to items in the search display that matched the WM stimulus, our findings suggest that it cannot be fully automatic, in the sense that guidance effects decreased when a secondary task (articulatory suppression) was introduced (cf. Humphreys, 1985; Shiffrin \& Schneider, 
1977). Rather, WM seems to act in an autonomous manner (Humphreys, 1985), once consolidated, but this was not independent of the resources available for the task.

\section{AUTHOR NOTE}

This work was supported by grants from the British Academy, the BBSRC, and the MRC (U.K.). We thank Carolina Bonivento for her help with data collection in Experiment 1. Correspondence concerning this article should be addressed to D. Soto, Imperial College London, Division of Neuroscience and Mental Health, Charing Cross Campus, St. Dunstan's Road, London W6 8RP, England (e-mail: d.soto@imperial.ac.uk).

\section{REFERENCES}

Allen, R. J., BADDEley, A. D., \& Hitch, G. J. (2006). Is the binding of visual features in working memory resource-demanding? Journal of Experimental Psychology: General, 135, 298-313.

Baddeley, A. D. (1986). Working memory. Oxford: Oxford University Press, Clarendon Press.

BADDELEY, A. [D.] (1992). Working memory. Science, 255, 556-559.

BADDELEY, A. [D.] (2003). Working memory: Looking back and looking forward. Nature Reviews Neuroscience, 4, 829-839.

Chelazzi, L., Duncan, J., Miller, E. K., \& Desimone, R. (1998). Responses of neurons in inferior temporal cortex during memory-guided visual search. Journal of Neurophysiology, 80, 2918-2940.

Chelazzi, L., Miller, E. K., Duncan, J., \& Desimone, R. (1993) A neural basis for visual search in inferior temporal cortex. Nature, 363, 345-347.

Coltheart, M. (1980). The persistences of vision. Philosophical Transactions of the Royal Society B, 290, 57-69.

Coltheart, M. (1983). Iconic memory. Philosophical Transactions of the Royal Society B, 302, 283-294.

Desimone, R. (1998). Visual attention mediated by biased competition in extrastriate visual cortex. Philosophical Transactions of the Royal Society $B, 353,1245-1255$.

Desimone, R., \& Duncan, J. (1995). Neural mechanisms of selective visual attention. Annual Review of Neuroscience, 18, 193-222.

DownING, P. E. (2000). Interactions between visual working memory and selective attention. Psychological Science, 11, 467-473.

DownING, P. E., \& DodDs, C. M. (2004). Competition in visual working memory for control of search. Visual Cognition, 11, 689-703.

DunCAN, J., Humphreys, G. W., \& Ward, R. (1997). Competitive brain activity in visual attention. Current Opinion in Neurobiology, 7, 255-261.

Humphreys, G. W. (1985). Attention, automaticity, and autonomy in visual word processing. In D. Besner, T. G. Waller, \& G. E. MacKinnon (Eds.), Reading research: Advances in theory and practice (Vol. 5, pp. 253-310). New York: Academic Press.

Jolicceur, P., \& Dell'ACQua, R. (1998). The demonstration of shortterm consolidation. Cognitive Psychology, 36, 138-202.
KARNATH, H.-O. (1988). Deficits of attention in acute and recovered visual hemi-neglect. Neuropsychologia, 26, 27-43.

Moores, E., Laiti, L., \& ChelazzI, L. (2003). Associative knowledge controls deployment of visual selective attention. Nature Neuroscience, 6, 182-189.

Olivers, C. N. L., Meijer, F., \& Theeuwes, J. (2006). Feature-based memory-driven attentional capture: Visual working memory content affects visual attention. Journal of Experimental Psychology: Human Perception \& Performance, 32, 1243-1265.

Psychology Software Tools (2002). E-Prime (Version 1.0) [Computer software]. Pittsburgh, PA: Author.

ShIFFrIN, R. M., \& SCHNEIDER, W. (1977). Controlled and automatic human information processing: II. Perceptual learning, automatic attending, and a general theory. Psychological Review, 84, 127-190.

Soto, D., Heinke, D., Humphreys, G. W., \& Blanco, M. J. (2005). Early, involuntary top-down guidance of attention from working memory. Journal of Experimental Psychology: Human Perception \& Performance, 31, 248-261.

Soto, D., \& Humphreys, G. W. (2006). Seeing the content of the mind: Enhanced awareness through working memory in patients with visual extinction. Proceedings of the National Academy of Sciences, 103, 4789-4792.

Soto, D., \& Humphreys, G. W. (2007). Automatic guidance of visual attention from verbal working memory. Journal of Experimental Psychology: Human Perception \& Performance, 33, 730-737.

Soto, D., Humphreys, G. W., \& Heinke, D. (2006a). Dividing the mind: The necessary role of the frontal lobes in separating memory from search. Neuropsychologia, 44, 1282-1289.

Soto, D., Humphreys, G. W., \& Heinke, D. (2006b). Working memory can guide pop-out search. Vision Research, 46, 1010-1018.

Theeuwes, J., Reimann, B., \& Mortier, K. (2006). Visual search for featural singletons: No top-down modulation, only bottom-up priming. Visual Cognition, 14, 466-489.

Vogel, E. K., Woodman, G. F., \& Luck, S. J. (2006). The time course of consolidation in visual working memory. Journal of Experimental Psychology: Human Perception \& Performance, 32, 1436-1451.

WARD, R., GoODrich, S., \& DrIVEr, J. (1994). Grouping reduces visual extinction: Neuropsychological evidence for weight-linkage in visual selection. Visual Cognition, 1, 101-129.

Woodman, G. F., \& LUCK, S. J. (2007). Do the contents of visual working memory automatically influence attentional selection during visual search? Journal of Experimental Psychology: Human Perception \& Performance, 33, 363-377.

\section{NOTE}

1. Soto et al. (2005) examined performance with a relatively lowsaliency target. Note, however, that the effects of WM guidance here were equally strong on low- and high-saliency targets, so this was unlikely to be a crucial factor in determining the null effect of the initial item when it did not have to be held in WM. 


\section{APPENDIX}

\section{Experiment 1: Analyses of the Fastest Responses}

We assessed whether similar effects were present in the fast end of the distribution of RTs (see Soto et al., 2005; Soto et al., 2006b). Analyses of the RTs up to the 5th percentile showed that RTs were slower on invalid than on neutral trials ( $780 \mathrm{vs} .739 \mathrm{msec})[F(1,9)=9.25, p<.014]$, performance was faster with high- than with low-saliency targets $(655$ vs. $865 \mathrm{msec})[F(1,9)=39.53, p<.0001]$, and RTs decreased as the ISI increased $(821,736$, and $722 \mathrm{msec}$ across the short, medium, and long ISIs) $[F(2,18)=11.5, p<.005]$. Analyses up to the 10th percentile showed similar effects $[F(1,9)=16.82, p<.003$, for validity; $F(2,18)=8.32, p<.013$, for ISI; and $F(1,9)=42.25, p<.0001$, for saliency].

\section{Experiment 2: Analyses of the Fastest Responses}

Analyses up to the 5 th and 10 th percentiles gave nonsignificant effects of validity $[F(1,14)<1$ and $F(1,14)=$ $1.62, p<.223]$.

\section{Experiment 3: Analyses of the Fastest Responses}

Analyses of the RTs up to the 5th percentile showed that performance was reliably slower on invalid in comparison with neutral trials $[F(1,13)=8.43, p<.012]$; in addition, RTs showed faster performance as the ISI increased $[F(2,26)=9.10, p<.001]$. This also held for RTs up to the 10th percentile $[F(1,13)=11.33, p<$ .005 , and $F(2,26)=8.43, p<.002$, respectively]. The interaction between validity and ISI was not reliable for both distribution analyses. 